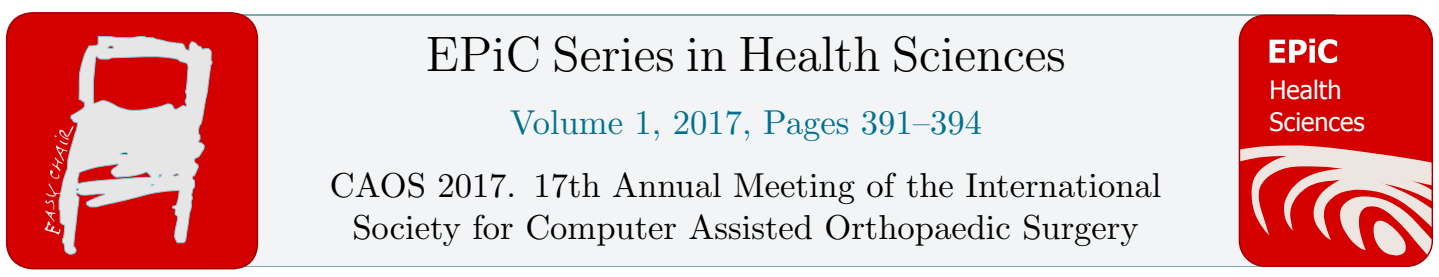

\title{
Knee Periprosthetic infections: CAOS use in one stage procedures.
}

\author{
Gerard Giordano $\mathrm{MD}_{1}$ * \\ Joseph Ducuing Hospital, Toulouse, 31027, France \\ ortho.giordano@gmail.com
}

\section{INTRODUCTION}

The functional and anatomical results of TKA revisions are less good than a primary TKA (1). The difficulties to adapt the preoperative planification after the component removal and to interpret the informations about the anatomical landmarks, the gap balancing, the management of the bone loss before and after the debridement are wellknow. On the other hand, the TKA revision frequency increases and we must improve our surgeries and prepare the next standard of these surgeries (5). The aim of this study was to evaluate the CAOS / one stage strategy to treat the knee PJIs $(2,3)$.

\section{MATERIALS}

Study design and patients

In this prospective study, between September 2011 and december 2014, 41 patients treated for chronic knee PJI in a one stage revision. An imageless CAOS system (ExactechGPS, Blue-Ortho, Gieres) was used with a personnalized profile. All surgeries were performed with the same protocole; a one stage procedure, independently of the type of germ, with no use of tourniquet, no drainage, by performing the same debridement procedure step by step and by using the same knee components. All operations were performed by a single senior surgeon.

Indications for the revision TKA were (1) revision of a primary TKA or unicondylar knee arthroplasty $(n=27)$ or (2) revision of revisionTKA $(n=15)$.

\section{Operative technique}

The Optetrak CC knee system (Exactech, Gainesville, FL) was implanted for all patients. All cases were performed under guidance of an imageless CAOS system (ExactechGPS, BlueOrtho, Gieres, FR) (Fig. 2). At the beginning of the surgery, the surgeon attached active trackers to the distal femur and proximal tibia. Then, the acquisition of the specific anatomical landmarks was performed. From this point, the surgeon targeted distal femoral and proximal tibial cuts perpendicular to the mechanical axis reconstructed from the previously acquired landmarks. Bone defects were assessed with the help of the CAOS 
system and cut heights were targeted to minimize bone loss as well as to optimize reconstruction height. Once all the cuts were completed, the length, angle, and diameter of the stem extensions were chosen in order to maintain mechanical alignment of the components. Finally, the trial components were put in place and the leg alignment in extension was checked by the CAOS system and the hip-knee-ankle (HKA) angle was recorded.

\section{Post-operative evaluation}

The measurement of the HKA angle on full-length weight bearing radiograph in the standing position, the Oxford score and the ROM were evaluated pre and post-operatively. The success of the infection healing was confirmed after minimally two years of follow-up.

\section{RESULTS}

27 males and 14 females with an average age of 71 years old (55-87) were treated for a PIJ (1 unicompartimental prosthesis, 26 TKA and 15 RTKA). The mean follow-up was 41 months (30 months - 6 years). The average time of surgery was $135 \mathrm{mn}$ (120-195) for an average hospitalisation duration of 10 days (7-16). No postoperative outliers were reported (mean preop.: $-1,6^{\circ}+l_{-}-5,1^{\circ}$ - Post-op. $-0,3^{\circ}+l_{-}-1,4^{\circ}$ ). The average ROM were $115^{\circ}$ (90$130^{\circ}$ ) (Fig. 1). The rate of success for the infection was $92,7 \%$. We report no specific CAS complications and all the navigations were finalized. In this series of complex cases, the rate of infection healing is $92,7 \%$.

\section{DISCUSSION}

The GPS system provided a safer and simplier procedure to optimize these complex situations, by a real time feedback and the immediate objective validations of the surgical steps (gap, axes, femoral rotation, cuts...). The bone reconstruction management, particularly after debridement, is reproducible and improved. The CAOS allows us to securise the Optetrak CC component implantation.

This first series initiated the " GPS RTKA » project to create a dedicated software. Since January 2016, 9 TKA revisions were performed with the new «RTKA » profile with immediate very good functional results and no complications.

With a rate of success of $92,7 \%$, the one stage option is valided. Using CAS is a safe option with no specific complication. It increases the quality of the ROM, a earlier functional recovery and a better middle term clinical result. Both combined, It should be a optimal medicoeconomical solution compared revision using mechanical ancillary. $(2,3,4)$

\section{REFERENCES}


1. Hamilton DF, Howie CR, Burnett R, Simpson AH, Patton JT. Dealing with the predicted increase in demand for revision total knee arthroplasty: challenges, risks and opportunities. Bone Joint J. 2015;97-B(6):723-8

2. Jenny, JY, Diesinger, Y. Navigated revision TKR: a comparative study with conventional instruments. Orthopedics 2010 Oct. 33:58-61.

3. Ochs, BG, Schreiner, AJ, de Zwart, PM, Stöckle, U, Gonser, CE. Computer-assisted navigation is beneficial both in primary and revision surgery with modular rotatinghinge knee arthroplasty. Knee Surg Sports Traumatol Arthrosc 2016 Jan. 24:64-73.

4. Massin, P, Boyer, P, Pernin, J, Jeanrot, C. Navigated revision knee arthroplasty using a system designed for primary surgery. Comput Aided Surg 2008 Jul. 13:179-87.

5. Sharkey PF, Hozack WJ, Rothman RH, Shastri S, Jacoby SM. Insall Award paper: why are total knee arthroplasties failing today? Clin Orthop Relat Res. 2002;(404): 7-13

\section{DISCLOSURES}

Gerard Giordano: Design surgeon consultant for Exactech, Inc.

Figure 1: Results: Range of motion 


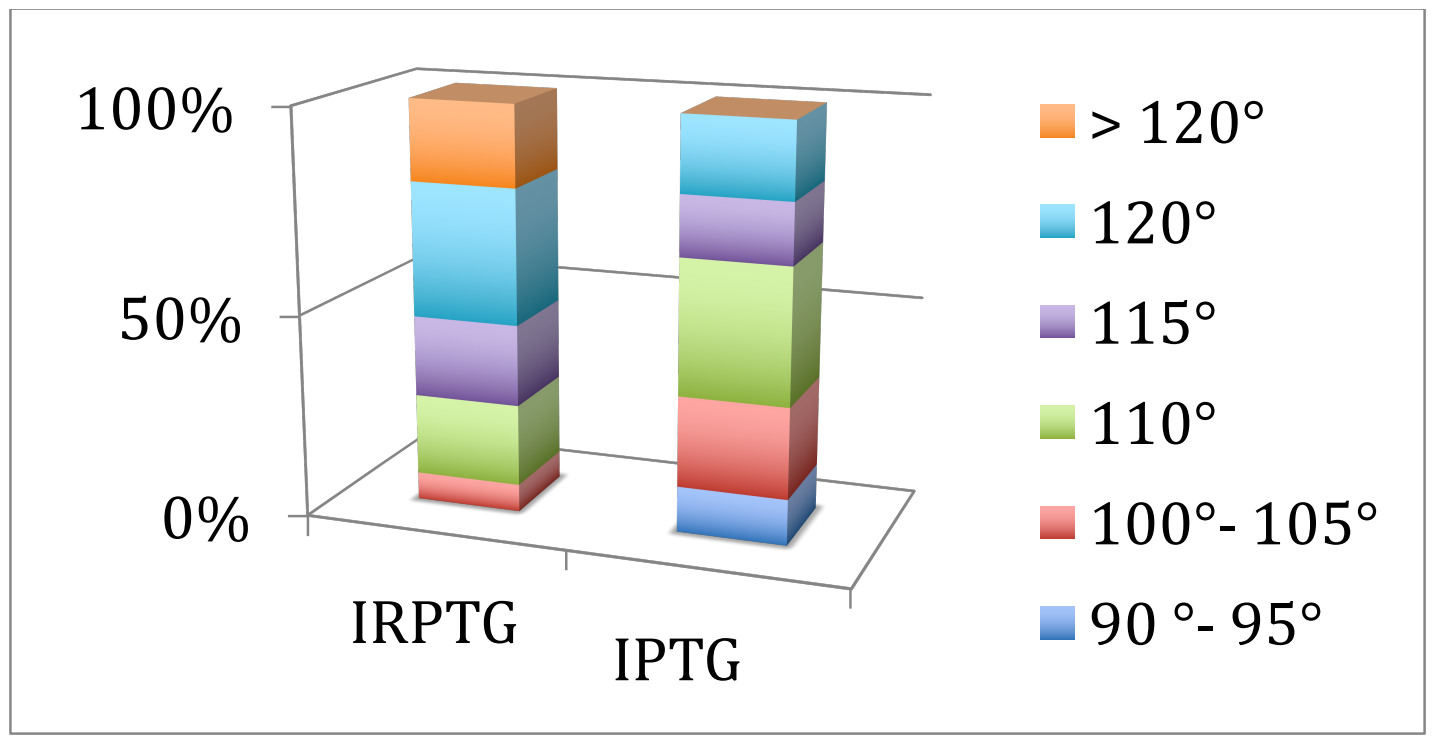

Figure 2: Oxford scores - Clinical outcomes stabilized between 6 months and one year

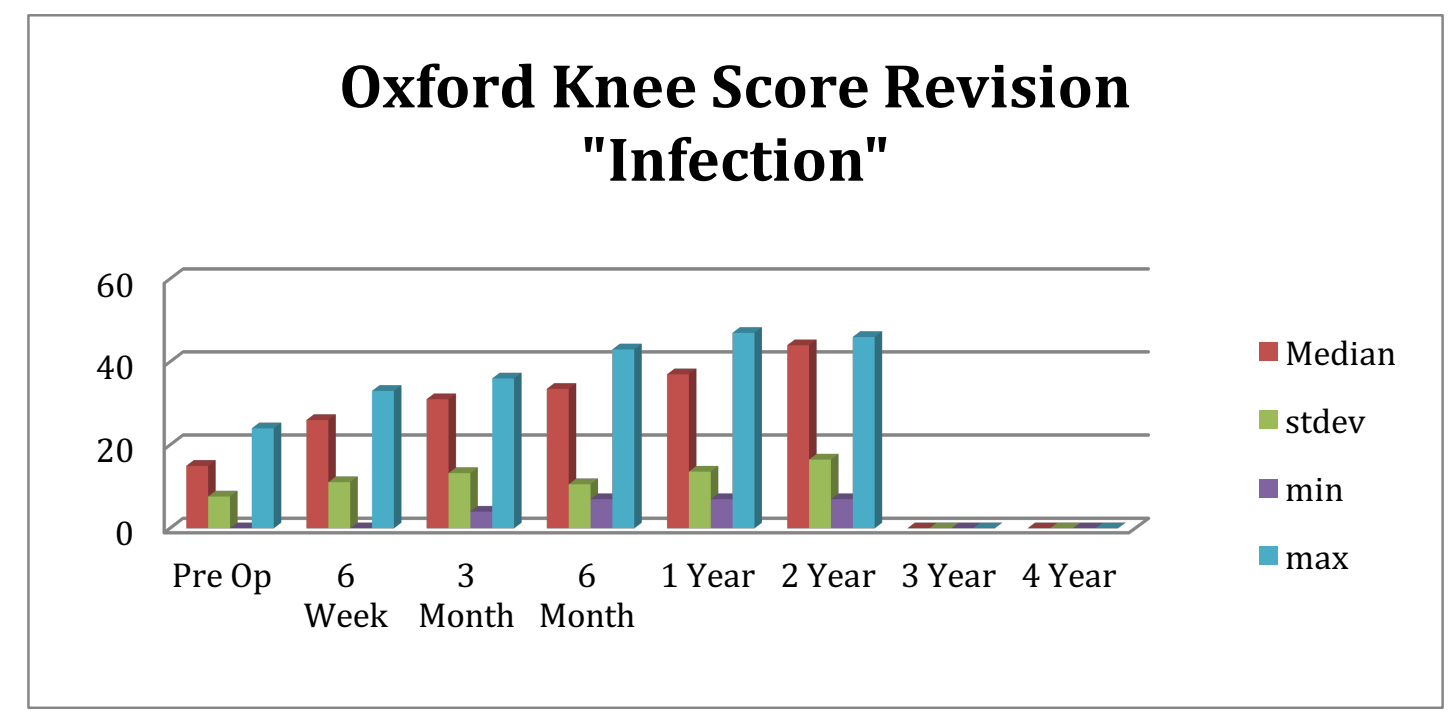

